Proceedings of the 1996 IEEE

International Conference on Robotics and Automation

Minneapolis, Minnesota - April 1996

\title{
Sensor Based Planning for a Planar Rod Robot
}

\author{
Howie Choset Joel Burdick \\ Division of Engineering and Applied Science \\ California Institute of Technology, Pasadena, CA 91125
}

\begin{abstract}
Sensor based planning for rod-shaped robots is necessary for the realistic deployment of noncircular symmetric robots into unknown environments. To this end, the rod hierarchical generalized Voronoi graph (rod-HGVG), introduced in this paper, is a roadmap for rod-like robots. A key feature of this roadmap is that it can be incrementally constructed using distance (range) information. This planning paradigm is an extension of previous work on sensor based planning for point robots.
\end{abstract}

\section{Introduction}

Sensor based planning makes use of sensor information reflecting the current state of the environment, in contrast to classical planning, which assumes full knowledge of the environment prior to planning. This paper extends the sensor based planning scheme for a point robot [3] to the case of robots which can be modeled as rods. This work is the next step towards the ultimate goal of sensor based planning for an articulated multi-body chain robot. The primary advantage of this method is that distance measurements are made entirely in the workspace, instead of the configuration space where measuring distance with conventional sensors is quite difficult.

The rod hierarchical generalized Voronoi graph (rodHGVG), introduced in this paper, is a roadmap which captures the global topological properties of the robot's free space and has the following important properties: accessibility, departability and connectivity. These properties imply that the planner can construct a path between any two points in a connected component of the robot's free space by first finding a path onto the roadmap (accessibility), traversing the roadmap to the vicinity of the goal (connectivity), and then constructing a path from the roadmap to the goal (departability).

\section{Relation to Prior Work}

Sensor based planning has received increased attention, as it is a requirement for realistic deployment of autonomous robots in unstructured environments. For a review of many sensor based planning techniques, see [13]. Unfortunately, current sensor based planning methods are limited because: (1) many are based on heuristic algorithms, and it is therefore impossible to prove if they will work in all possible environments; (2) proof of convergence for other algorithms is limited to the case of a point in two-dimensional environments (for example, Lumelsky's "bug" algorithm [7]); or (3) the robot is assumed to be a point in configuration space, where its sensors can measure distance in configuration space. The goal of this work is to develop provably correct rod motion planning schemes which can be robustly implemented with realistic sensors.

The generalized Voronoi diagram (GVD) is a roadmap which was first used for motion planning in [12]. Active research in applying Voronoi diagrams to motion planning began with Ó'Dúnlaing and Yap's work [8], which considered motion planning for a disk in the plane. However, the method in [8] requires full knowledge of the world's geometry prior to the planning event. This work was extended to the case where the robot is a rod in [9], but it, too, requires full knowledge of the world's geometry prior to the planning event. Recently, Cox and Yap [5] developed an "on-line" strategy for path planning for rods. Although this method can be readily modified with tactile sensors for sensor based use, it does not provide a roadmap of the rod robot's free space. The goal of the work described in this paper is to define a roadmap for a rod, and demonstrate that it can be constructed using realistic sensors.

An incremental approach to creating a Voronoi diagram-like structure, which is limited to circular robots in the plane, is introduced in [10]. To our knowledge, the only endeavors pertaining to sensor based adaptations of roadmaps for configuration space dimensions greater than two are [11] (which is based on Canny and Lin's Opportunistic Path Planner (OPP) [1]), and [3] (which is where the generalized Voronoi graph is defined). A limitation of these roadmaps is that distance measurements are assumed to be made in a configuration space (or some parameterization of it). In this paper, we define a configuration space roadmap based on workspace distance measurements which are obtainable from realistic sensors. Therefore, the rod-HGVG is easier to construct using realistic sensors.

This paper is an extension of a previous sensor based planning scheme which is based on the generalized Voronoi graph (GVG) [3]. The GVG is the foundation for a point robot roadmap. In this paper, we term the GVG the point GVG in order to distinguish it from the generalized Voronoi graph for a rod.

The point GVG was defined in terms of a distance 


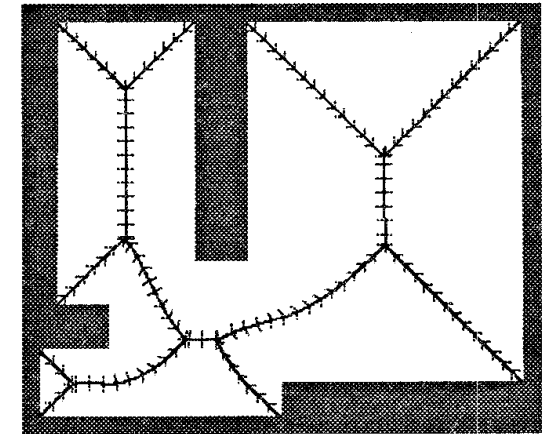

Fig. 1. The ticked line segments form the planar GVG for a bounded environment. The ticks point at the nearest point on an obstacle, and are thus the negated gradients of the distance function.

function

$$
d_{i}(r)=\min _{c \in C_{i}}\|r-c\|
$$

where $r \in \mathbb{R}^{2}$ and $C_{i}$ is a convex obstacle. The basic building block of the point GVG is the two-equidistant surjective surface which is a set of points equidistant to two convex obstacles, and is denoted

$S S_{i j}=\left\{r \in \mathbb{R}^{m}:\left(d_{i}-d_{j}\right)(r)=0\right.$ and $\left.\nabla d_{i}(r) \neq \nabla d_{j}(r)\right\}$, where $\nabla d_{i}(r)$ is a unit vector based at $r$ and pointing away from $c$ along a line defined by $c$ and $r$. In constructing the GVG, we are interested in a subset of $S S_{i j}$ termed the two-equidistant face which is defined as

$$
\mathscr{F}_{i j}=\left\{r \in \operatorname{cl}\left(S S_{i j}\right): d_{i}(r) \leq d_{h}(r) \forall h\right\} .
$$

The intersection of $\mathcal{F}_{i j}$ and $\mathcal{F}_{j k}$ forms a three-equidistant face, denoted $\mathcal{F}_{i j k}$, and it is the set of points equidistant to three obstacles: $C_{i}, C_{j}$, and $C_{k}$ [3].

In the plane, the two-equidistant faces and threeequidistant faces are one and zero-dimensional, respectively and the point GVG is the collection of these onedimensional edges and zero-dimensional vertices. In the plane, the GVG, which is the set of points equidistant to two or more obstacles, is always connected. See Figure 1. In $\mathbb{R}^{m}$, the GVG is the set of points equidistant to $m$ obstacles and is always one-dimensional, though not necessarily connected. [3].

\section{Rod Distance Function}

Definition 3.1 (Rod) A rod $R$ is a line segment of length $L$ that has two end points $P$ and $Q$.

The configuration space of the rod is $S E(2)(S E(2) \simeq$ $\left.\mathbb{R}^{2} \times S^{1}\right)$. Let $q$ be the configuration of the rod, and let it be determined by the $x$ and $y$ coordinates of the point $P$, and the orientation of the rod with respect to the horizontal, i.e. $q=(x, y, \theta)$. See Figure 2. Let $q(P)$ be

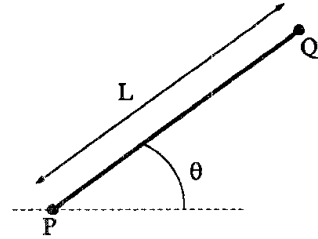

Fig. 2. The configuration of a rod is determined by the $x$ and $y$ coordinates of $P$ and the orientation of the rod with respect to the horizontal.

the $x$ and $y$ coordinates of the point $P$ when the rod is at configuration $q$, let $q(\theta)$ be the orientation of the rod when it is at configuration $q$, and let $q(R)$ be the set of points in the plane that the rod occupies when it is at configuration $q$. Note that $q(P) \in \mathbb{R}^{2}, q(\theta) \in S^{1}$, and $q(R) \subset \mathbb{R}^{2}$. Let superscripts ${ }^{x}$ and ${ }^{y}$, denote the $x$ and $y$ coordinates, respectively, of a point in the plane. For example, $q(P)^{x}$ is the $x$ coordinate of the point $P$ at configuration $q$.

A function which encodes the distance between the robot and nearby obstacles is key to our definitions. We assume a rod robot $R$ is operating in a subset $\mathcal{W}$ of $\mathbb{R}^{2} . \mathcal{W}$ is populated by obstacles $C_{1}, \ldots, C_{n}$ which are convex sets. Non-convex obstacles are modeled as the union of convex shapes. It is assumed that the boundary of $\mathcal{W}$ is a collection of convex sets, which are members of the obstacle set $\left\{C_{i}\right\}$.

\section{Definition 3.2 (Rod Single Object Distance)}

The rod single object distance function is the distance between an obstacle, $C_{i}$, and a $\operatorname{rod}, R$, when the rod is at a configuration $q$. It is determined by

$$
\begin{aligned}
D_{i}(q) & =\min _{r \in q(R), c \in C_{i}}\|r-c\|, \\
& =\min _{l \in[0, L], c \in C_{i}}\left\|\left[\begin{array}{l}
q(P)^{x}+l \cos (\theta)-c^{x} \\
q(P)^{y}+l \sin (\theta)-c^{y}
\end{array}\right]\right\| .
\end{aligned}
$$

An important characteristic of $D_{i}(q)$ is that it can be readily computed from sensor measurements made in the workspace. For example, the rod robot in Figure 3 has range sensors distributed around its perimeter. The distance between the obstacle and the rod is the measurement of the range sensor associated with a local minima of measurements. It can be shown that the roddistance function is continuous and smooth in the interior of the workspace for convex sets. Let the gradient of rod distance function, $\nabla D_{i}(q)$, be the $3 \times 1$ unit vector $\left[\nabla d_{i}(r) 0\right]^{T}$, where $r$ is the closest point on the rod $R$ to obstacle $C_{i}, \nabla D_{i}(q)$ describes the direction along which the rod must translate (with a fixed orientation) to maximally increase its distance from $C_{i}$. For convex sets, $\nabla D_{i}(q)$ is continuous. 


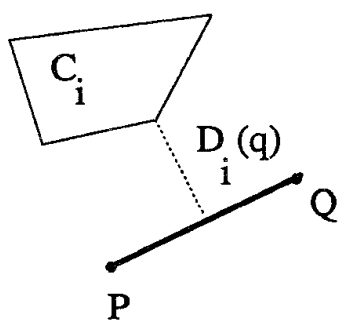

Fig. 3. The distance from the rod (thick solid line) to an obstacle is the distance (dotted line) between the nearest point on the rod to the obstacle and the nearest point on the obstacle to the rod.

\section{Rod-GVG}

The rod-GVG serves as a basis for the rod-HGVG. A key feature of the rod-GVG is that it is defined in terms of a distance function. Before defining the rod-GVG, we define the rod-GVD because the rod-GVG is an extension of the rod-GVD. (The rod-GVD was termed the Voronoi complex in [9]). The basic building block of the rod-GVD and the rod-GVG is the set of rod configurations equidistant to two sets $C_{i}$ and $C_{j}$, which we term the configuration two-equidistant surface,

$$
\mathrm{eS}_{i j}=\left\{q \in S E(2): D_{i}(q)=D_{j}(q)>0\right\} .
$$

Of particular interest is the subset of $\mathcal{E S}_{i j}$ termed the configuration two-equidistant surjective surface,

$$
\operatorname{eSS}_{i j}=\left\{q \in \operatorname{eS}_{i j}: \nabla D_{i}(q) \neq \nabla D_{j}(q)\right\} .
$$

which is the set of configurations, $q$, that are equidistant to two objects such that $\nabla D_{i}(q) \neq \nabla D_{j}(q)$ (i.e., the function $\left(D_{i}-D_{j}\right)(q)$ is surjective). This definition is required to deal with non-convex sets that are modeled as the finite union of convex sets.

The configuration two-equidistant face,

$$
\begin{aligned}
\mathcal{E F}_{i j}=\left\{q \in \operatorname{cl}\left(\mathcal{C S S}_{i j}\right):\right. & \\
& \left.D_{i}(q)=D_{j}(q) \leq D_{h}(x) \quad \forall h \neq i, j\right\},
\end{aligned}
$$

is the set of configurations equidistant to obstacles $C_{i}$ and $C_{j}$, such that each point $x$ in $\operatorname{cl}\left(\operatorname{CSS}_{i j}\right)$ is closer to $C_{i}$ and $C_{j}$ than any other obstacle. See Figure 4 for examples of rods whose configurations are in the configuration two-equidistant face. The configuration two-Voronoi set, $\mathrm{eF}^{2}$, is the union of all configuration two-equidistant faces, i.e.,

$$
\mathbf{e F}^{2}=\bigcup_{i=1}^{n-1} \bigcup_{j=i+1}^{n} \mathcal{e F}_{i j}
$$

Let the rod generalized Voronoi diagram (rod-GVD) be $\mathbf{e F}^{2}$, which is the set of configurations in which the rod is equidistant to two or more closest points on the boundary of a bounded space.

Accordingly, one can define a configuration threeequidistant face and configuration three-Voronoi set re-

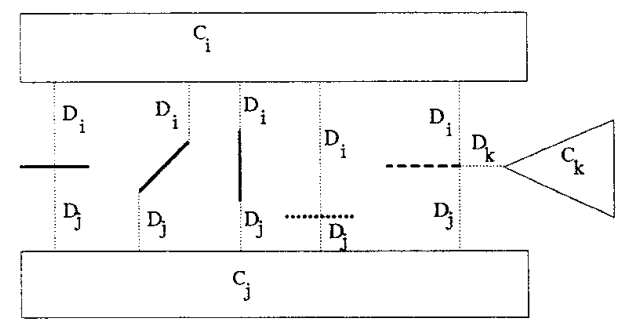

Fig. 4. The thick solid lines are rods which are in the configuration two-equidistant face defined by obstacles $C_{i}$ and $C_{j}$. The light dotted lines delineate the distance to the nearest obstacle. The thick dotted line is an example of a rod which is not in a configuration two-equidistant face because it is closer to $C_{j}$ than $C_{i}$. The thick dashed line is not in the two-equidistant face either because it is closer to $C_{k}$.

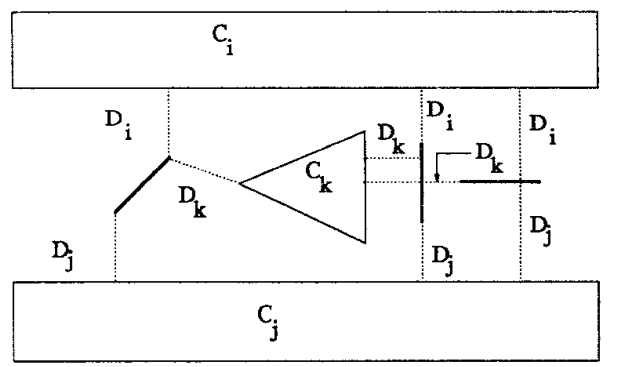

Fig. 5. The thick solid lines represent three configurations of the rod whose configurations are in the configuration threeequidistant face defined by obstacles $C_{i}, C_{j}$ and $C_{k}$. The thin dotted lines represent the distance between the rods and an obstacles.

spectively as

$$
\begin{aligned}
\mathfrak{e F}_{i j k} & =\mathcal{e F}_{i j} \cap \mathcal{E F}_{i k}, \\
\mathfrak{E F}^{3} & =\bigcup_{i=1}^{i=m-2} \bigcup_{j=i+1}^{j=m-1} \bigcup_{k=j+1}^{k=m} \mathcal{e F}_{i j k} .
\end{aligned}
$$

These structures are the set of configurations where the rod is equidistant to three obstacles. See Figure 5. Next, the configuration four-equidistant face and configuration four-Voronoi set are defined respectively as

$$
\begin{aligned}
\mathrm{eF}_{i j k l} & =\mathrm{eF}_{i j} \cap \mathcal{E F}_{i k} \cap \mathcal{C F}_{i l} \\
\mathcal{C F}^{4} & =\bigcup_{i=1}^{i=m-3} \bigcup_{j=i+1}^{j=m-2} \bigcup_{k=j+1}^{k=m-1} \bigcup_{l=k+1}^{l=m} \mathrm{eF}_{i j k}(6)
\end{aligned}
$$

Finally, in keeping with the generalized Voronoi graph literature, the configuration three equidistant faces are also termed rod-GVG edges and the configuration four equidistant faces are termed rod-GVG vertices. Using the pre-image theorem, it can be shown that the rod-GVG edges and rod-GVG vertices are one and zero dimensional, respectively. Now, we can define the rod-GVG.

Definition 4.1 (RoD-GVG) The collection of rodGVG edges and of rod-GVG vertices is the rod-GVG.

The rod-GVG edges can be incrementally constructed using only line of sight information in a fashion similar to the incremental construction of point 


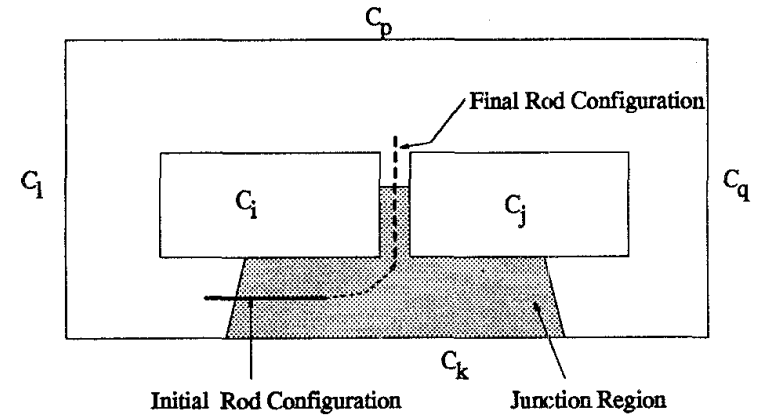

Fig. 6. The rod may pass from its initial configuration to its final configuration while staying inside of the junction region defined by $C_{i}, C_{j}$, and $C_{k}$.

GVG edges described in [4]. Just like the point GVG edges, the rod-GVG edges are traced in an incremental manner using an adaptation of numerical continuation techniques [6] which trace the roots of the expression $G(y, \lambda)=0$ as the parameter $\lambda$ varies where $q=(y, \lambda)$.

$$
G(y, \lambda)=\left[\begin{array}{l}
D_{i}(y, \lambda)-D_{j}(y, \lambda) \\
D_{i}(y, \lambda)-D_{k}(y, \lambda)
\end{array}\right]
$$

If $G$ is surjective (whose proof is omitted due to space restrictions), then the implicit function theorem implies that the roots of $G(y, \lambda)$ locally define a rod-GVG edge as $\lambda$ is varied. Since the incremental construction procedure is defined in terms of the distance function, it is amenable to sensor based implementation.

\section{Accessibility}

Accessibility is the property that the rod can move from any configuration in the workspace to a configuration on a rod-GVG edge. The accessibility algorithm described below prescribes a path to a rod-GVG edge such that the rod moves with a fixed orientation. In a sense, this reduces the problem to accessibility of a point in a planar configuration space because the configuration space of a rod with a fixed orientation is $\mathbb{R}^{2}$.

For the study of accessibility and connectivity, we define the junction region, $\partial_{i j k}$, to be the set of points where obstacles, $C_{i}, C_{j}$, and $C_{k}$ are the three closest line of sight obstacles. That is,

$$
\begin{gathered}
\partial_{i j k}=\left\{q \in S E(2): D_{i}(q) \leq D_{h}(q), D_{j}(q) \leq D_{h}(q),\right. \\
\text { and } \left.D_{k}(q) \leq D_{h}(q) \forall h \notin\{i, j, k\}\right\} .
\end{gathered}
$$

It can be shown that $\mathcal{C F}_{i j k} \subset \partial_{i j k}$ and that the union of all the junction regions are all the configurations of the rod robot in the workspace. Figure 6 illustrates an example of a junction region.

The following proposition demonstrates that there exists a path from a configuration in a junction, $\partial_{i j k}$, to a configuration on $\mathrm{eF}_{i j k}$. For the following proposition, let $\pi_{T_{q}}$ eF $F_{i j}$ be the operator which projects a vector onto the tangent space of $\mathcal{C F}_{i j}$. For example, $\pi_{T_{q}} \mathcal{e F}_{i j} \nabla D_{i}(q)$

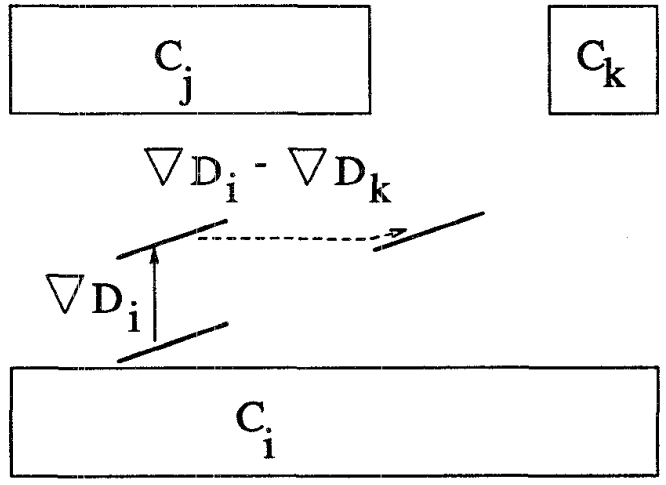

Fig. 7. The solid lines represent the rod, which is moving with a fixed orientation from an initial configuration to the rodGVG. The solid arrow represents the portion of the path in which the rod is following the gradient to the nearest obstacle. The dotted arrow corresponds to the final portion of the path where the rod moves toward $C_{k}$ while remaining equidistant to $C_{i}$ and $C_{j}$ (i.e., the rod follows $\pi_{T_{q}}$ ef $_{i j} \nabla D_{i}(q)-$ $\left.\pi_{T_{q}} \mathrm{eF}_{i, j} \nabla D_{k}(q)\right)$.

is the gradient vector, $\nabla D_{i}(q)$, projected onto the tangent space of $\mathrm{CF}_{i j}$.

Proposition 5.1 (Rod Accessibility) Let the convex obstacles $C_{i}, C_{j}$ and $C_{k}$ be the three closest obstacles to a rod, $R$, at an initial configuration, $q \in \partial_{i j k}$, such that $D_{i}(q)<D_{j}(q)<D_{k}(q)$, and $C_{i}, C_{j}$ and $C_{k}$ are within line of sight of the rod. There exists a path from the initial configuration $q$ to the rod-GVG edge, $\mathcal{C} \mathcal{F}_{i j k}$ in the following two steps: (1) while maintaining a fixed orientation, the rod moves away from the nearest point on $C_{i}$ (i.e., it traces a path following $\nabla D_{i}$ ) until the rod is doubly equidistant to $C_{i}$ and $C_{j} ;(2)$ then, while maintaining double equidistance to $C_{i}$ and $C_{j}$ the rod moves towards $C_{k}$ by following the difference of the gradients $\nabla D_{i}$ and $\nabla D_{k}$ projected onto $\mathcal{C F}_{i j}$ (i.e., $\left.\pi_{T_{q} \text { eF }_{i j}} \nabla D_{i}(q)-\pi_{T_{q} \mathcal{E F}_{i j}} \nabla D_{k}(q)\right)$ until it is triply equidistant to $C_{i}, C_{j}$ and $C_{k}$.

The accessibility path is defined in terms of distance gradients to the nearest obstacles, and is thus already implementable for sensor based use [2]. See Figure 7 for an example of the rod accessing a point on a rodGVG edge. The proof of this proposition appears in the Appendix.

COROLLARY 5.2 There exists a continuous map $H: \partial_{i j k} \times[0,1] \rightarrow \partial_{i j k}$ where $H\left(q_{a}, t\right)$ describes the rod accessibility path starting at a configuration, $q_{a} \in \partial_{i j k}$, such that $H\left(q_{a}, 0\right)=q_{a}, H\left(q_{a}, .5\right)=q_{b}$ and $H\left(q_{a}, 1\right)=q_{c}$ where $q_{b} \in \mathcal{C} \mathcal{F}_{i j}$ and $q_{c} \in \mathcal{C F}_{i j k}$. M[oreover, for all $t \in[0,1], H\left(q_{a}, t\right)(\theta)=q_{a}(\theta)$ (i.e., the rod's orientation remains fixed).

The proof of this proposition appears in the Appendix. 


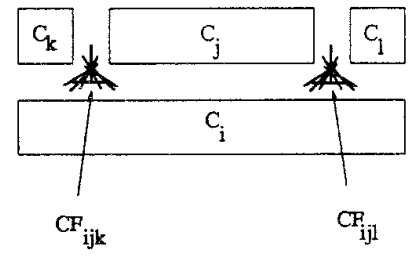

Fig. 8. The two clusters of solid lines represent rods whose configurations are triply equidistant to three obstacles. The left. cluster represents rods whose configurations are elements of the rod-GVG edge $\mathcal{C} \mathcal{F}_{i j k}$, and the right cluster are elements of $\mathcal{E F}_{i k l}$. In this example, both rod-GVG edges are diffeomorphic to $S^{\mathbf{1}}$ (i.e., they are cyclic) and neither rod-GVG edge is connected to any other rod-GVG edge.

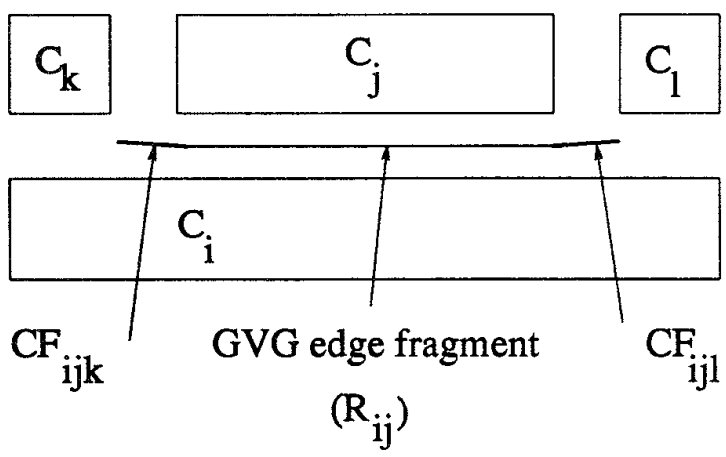

Fig. 9. The dark line segment on the left represents a rod configuration in $\mathrm{CF}_{i j k}$, and the dark segment on the right represents a rod configuration in $\mathrm{eF}_{i j l}$. These rods are connected by the point GVG edge $\mathcal{F}_{i j}$. The point GVG edge gives rise to a linking structure termed the $R$-edge which connects $\mathcal{C F}_{i j k}$ and $\mathrm{eF}_{i j l}$.

\section{The Rod-HGVG}

It was shown in [9] that the configuration rod-GVD is connected. However, the rod-GVG is not necessarily connected as can be seen in Figure 8. In order to connect the rod-GVG, we define additional structures, termed $R$-edges, which link disconnected rod-GVG edges by exploiting the property that the point $G V G$ is connected in the plane. See Figure 9. The $R$-edges are the set of rod configurations which are tangent to the point GVG edge, in a specific fashion defined below. See Figure 11.

Recall that the tangent space of a planar point GVG edge is the line orthogonal to the line segment which connects the nearest points of the two nearest obstacles which locally define the point GVG edge [4]. Let $C_{i}(r)$ be the closest obstacle to a point $r$ in the plane. In this vein, let $c_{i}$ be the vector which connects $r$ and the closest point to $r$ on the closest obstacle $C_{i}(r)$.

We define a mapping $\beta$ which describes the tangent space of a point GVG edge at a point. Let $\beta: S \mathcal{S}_{i j} \rightarrow S^{1}$ be defined as

$$
\beta(r)=\operatorname{arctangent}\left(c_{i}(r)-c_{j}(r)\right)+\frac{\pi}{2},
$$

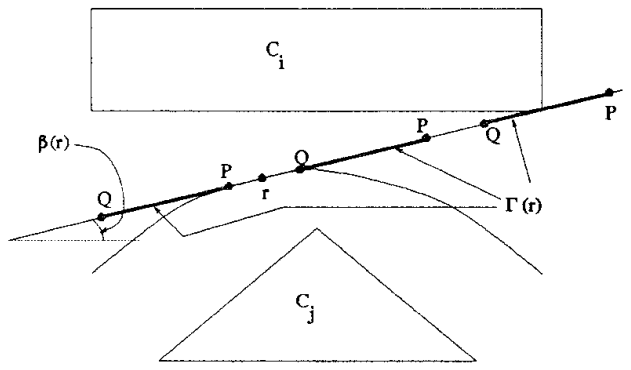

Fig. 10. The solid lines delineate three configurations of a rod that lie in $\Gamma(r) . \beta(r)$ is the angle which describes the tangent space to the point GVG edge at the point $r$.

where $\frac{\pi}{2}$ is measured in radians. It can be shown that $c_{i}(r)$ is a continuous function for convex sets [8], and thus $\beta$ is a continuous function.

Let the mapping $\Gamma: S S_{i j} \rightarrow S E(2)$ be defined as

$$
\Gamma(r)=\left\{\begin{array}{l}
r^{x}+l \cos (\beta(r)) \\
r^{y}+l \sin (\beta(r)): l \in \mathbb{R}^{1} \\
\beta(r)
\end{array}\right\}
$$

It can easily be seen that $\Gamma$ is a continuous mapping. $\Gamma(r)$ can be viewed as all the rods which lie in the tangent space of a two-equidistant surjective surface (and thus a two-equidistant face) at a point $r$. See Figure 10 for an example of $\Gamma(r)$.

Let the $R$-two-equidistant surjective surface defined by $C_{i}$ and $C_{j}$ be

$$
R S S_{i j}=\left\{\Gamma(r): r \in S S_{i j}\right\} .
$$

Since $R S S_{i j} \simeq S S_{i j} \times \mathbb{R}^{1}$, the dimension of $R S S_{i j}$ is two (recall that in $\mathbb{R}^{2}$, the dimension of $S S_{i j}$ is one [3]). $R S \mathcal{S}_{i j}$ may be viewed as (but is not) a tangent bundle of $S S_{i j}$.

Let the R-two-equidistant face be the set of configurations equidistant to two obstacles such that (I) there exists a point, $r \in R$, that is closer to obstacles $C_{i}$ and $C_{j}$ than any other point on the rod and (II) no other obstacle is closer to the rod than the two equidistant obstacles. In other words,

$$
\begin{array}{r}
R \mathcal{F}_{i j}=\left\{q \in \operatorname{cl}\left(R S S_{i j}\right): \text { such that } \exists r \in q(R)\right. \\
(\mathbf{I}) \mathrm{d}_{\mathrm{i}}(\mathrm{r}) \leq \mathrm{d}_{\mathrm{i}}\left(\mathrm{r}_{1}\right) \forall \mathrm{r}_{1} \in \mathrm{q}(\mathrm{R}) \text { and } \\
\left.(\mathbf{I I}) \mathrm{d}_{\mathrm{i}}(\mathrm{r}) \leq \mathrm{D}_{\mathrm{h}}(\mathrm{q}) \forall \mathrm{h} \neq \mathrm{i}, \mathrm{j}\right\} .
\end{array}
$$

In $S E(2)$, an $R$-two-equidistant face is termed an $R$ edge, (denoted $\mathcal{R}_{i j}$ ) because it is one-dimensional, as shown by the following proposition.

The inequality I $d_{i}(r) \leq d_{i}\left(r_{1}\right) \forall r_{1} \in q(R)$ determines how the rod is tangent to the point GVG edge. Let $r_{\min }$ be the point in $\mathcal{F}_{i j}$ where the distance to $C_{i}$ and $C_{j}$ is the smallest (i.e., for all $r \in \mathcal{F}_{i j} \backslash\left\{r_{\min }\right\}$, $\left.d_{i}(r)>d_{i}\left(r_{\min }\right)\right)$. For all points $r \in \mathcal{F}_{i j} \backslash\left\{r_{\min }\right\}$, the rod is tangent to the point GVG edge at $P$ or $Q$. Otherwise at $r_{\min }$, the rod is free to slide along the tangent 


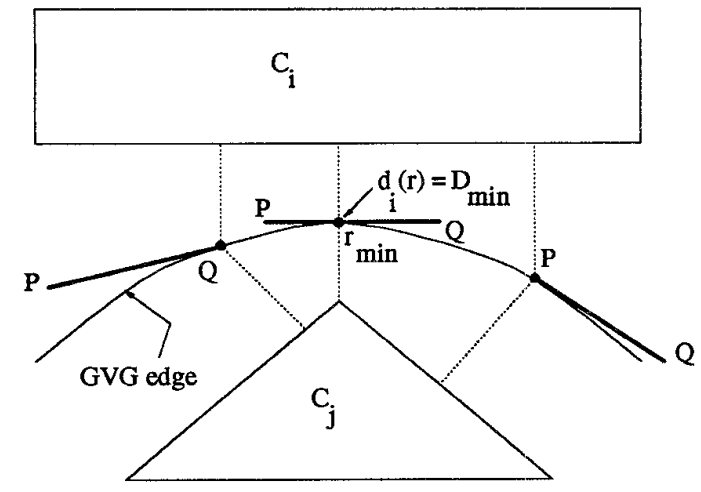

Fig. 11. The rod is moving from left to right while remaining tangent to the point GVG edge defined by obstacles $C_{i}$ and $C_{j}$. The thick solid lines represent different configurations of the rod in an $R$-edge. The dotted lines represent the shortest distance between the rod and the nearby obstacles. Note, for all configurations where the rod is not tangent to $r$ the closest point on the rod to $C_{i}$ and $C_{j}$ is either $P$ or $Q$.

space of the point GVG edge. See Figure 11.

Proposition 6.1 The $R$-edges are one dimensional in $S E(2)$.

The proof appears in the appendix.

The incremental construction technique of the $R$ edges is the same as the incremental construction procedure for point GVG edges (described in [4]), which is amenable to sensor based implementation. Hence, the $R$-edges can be constructed in an incremental fashion using only line of sight information.

DEFinition 6.2 (RoD-HGVG) The rod hierarchical generalized Voronoi graph (rod-HGVG) is the collection of rod-GVG edges and $R$-edges.

The following two lemmas, whose proofs appear in the appendix, indicate that a linking strategy using the $R$-edges echos the linking strategy defined by the second order GVG for the point GVG in higher dimensions [3].

LEMMA 6.3 The $R$-edges are subsets of configuration two-equidistant faces.

LEMMA 6.4 For all configurations $q \in \mathcal{R}_{i j}$, the rod does not intersect any obstacle (with the exception of points $P$ or $Q$ lying on the intersection of two obstacles).

\section{Connectivity of the rod-HGVG}

Proposition 7.1 Let $q_{1}$ and $q_{2}$ be two configurations of the rod. There exists a path between $q_{1}$ and $q_{2}$ if and only if there exists a path on the rod-HGVG between $H\left(q_{1}, 1\right)$ and $H\left(q_{2}, 1\right)$ where $H$ is the function which describes the accessibility path of the rod from an initial configuration to a configuration on the rod-GVG.

Proof: First we show the converse of this statement. By Proposition 5.1 and Corollary 5.2, there exists a path between $q_{1}$ and $H\left(q_{1}, 1\right)$, and there exists a path between $q_{2}$ and $H\left(q_{2}, 1\right)$. If there exists a path from $H\left(q_{1}, 1\right)$ to $H\left(q_{2}, 1\right)$ on the rod-HGVG, then there exists a path between $q_{1}$ and $q_{2}$.

Next, we show that if there exists a path between $q_{1}$ and $q_{2}$, then there exists a path between $H\left(q_{1}, 1\right)$ and $H\left(q_{2}, 1\right)$ on the rod-HGVG. If $q_{1} \in \mathcal{J}_{i j k}$ and $q_{2} \in \partial_{p q r}$ and there exists a path between them, then there exists a series of adjacent junction regions, $\partial_{i j k}, \partial_{i j l}, \ldots, \partial_{p q r}$ through which this path passes.

The problem of connectivity is now reduced to demonstrating that: (1) there exists a path between two rod-GVG edges in adjacent junction regions if and only if there exists an $R$-edge which links the two rod-GVG edges and (2) if two $R$-edges intersect a configuration three-equidistant face, $\mathcal{C F}_{i j k}$, then there exists a path between the two edges if and only if there exists a path between the two edges on $\mathrm{CF}_{i j k}$.

LEMMA 7.2 Let $\hat{q}_{1}, \hat{q}_{2}$ be two configurations in a junction region $\partial_{i j k}$. $\hat{q}_{1}$ and $\hat{q}_{2}$ are path connected within a junction, $\partial_{i j k}$, if and only if $H\left(\hat{q}_{1}, 1\right)$ and $H\left(\hat{q}_{2}, 1\right)$ are path connected in $\mathcal{C F}_{i j k}$.

Proof: By definition, $\mathcal{C F}_{i j k} \subset J_{i j k}$. By Proposition 5.1 and Corollary 5.2, there exists a path between $\hat{q}_{1}$ and $H\left(\hat{q}_{1}, 1\right)$, and there exists a path between $\hat{q}_{2}$ and $H\left(\hat{q}_{2}, 1\right)$. Therefore, if there exists a path between $H\left(\hat{q}_{1}, 1\right)$ and $H\left(\hat{q}_{2}, 1\right)$ in $\mathcal{C} \mathcal{F}_{i j k}$, then there exists a path between $\hat{q}_{1}$ and $\hat{q}_{2}$ in $\partial_{i j k}$.

Recall from Corollary 5.2 that there exists a continuous function, $H(q, t)$ which describes the accessibility for the rod.

Let $s(t)$ be a continuous function which describes a path from $\hat{q}_{1}$ to $\hat{q}_{2}$ such that $s(0)=\hat{q}_{1}$ and $s(1)=\hat{q}_{2}$. For all $t \in[0,1], H(s(t), 1) \in \mathcal{C F}_{i j k}$. The image of the path between $\hat{q}_{1}$ and $\hat{q}_{2}$ under $H(s(t), 1)$ is a connected path on $\mathcal{C F}_{i j k}$ because the image of a connected set under a continuous mapping is a connected set.

Now, it needs to be shown that the $R$-edges connect the rod-GVG edges in adjacent junction regions. The following proposition guarantees that there exists a path between two adjacent rod-GVG edges if and only if there exists a connected $R$-edge linking them.

LEMMA 7.3 Let $\bar{q}_{1} \in \mathrm{eF}_{i j k}$, and $\bar{q}_{2} \in \mathrm{eF}_{i j l}$ such that $\bar{q}_{1}$ and $\bar{q}_{2}$ are also on an $R$-edge, $\mathcal{R}_{i j}$, and $\mathcal{E} \mathcal{F}_{i j k}$ and $\mathcal{C} \mathcal{F}_{i j l}$ are in adjacent junctions regions. $\bar{q}_{1}$ and $\bar{q}_{2}$ are path connected if and only if the $R$-edge between them is connected.

Proof: If $\bar{q}_{1}$ and $\bar{q}_{2}$ lie on a connected $R$-edge then there exists a path between $\bar{q}_{1}$ and $\bar{q}_{2}$.

If there exists a path between $\bar{q}_{1}$ and $\bar{q}_{2}$, then there exists a point based GVG edge, $\mathcal{F}_{i j}$, which connects $\left[\bar{q}_{1}^{x}, \bar{q}_{1}^{y}\right]^{T}$ and $\left[\bar{q}_{2}^{x}, \bar{q}_{2}^{y}\right]^{T}$ in the plane. The $R$-edge which 
connects $\bar{q}_{1}$ and $\bar{q}_{2}$ is the image of a connected subset of $\mathcal{F}_{i j}$, which connects $\left[\bar{q}_{1}^{x}, \bar{q}_{1}^{y}\right]^{T}$ and $\left[\bar{q}_{2}^{z}, \bar{q}_{2}^{y}\right]^{T}$, under $\Gamma$. The $R$-edge is a connected set because the image of a connected set under a continous function is a connected set. Lemma 6.4 guarantees that all configurations of the rod on the $R$-edge do not intersect any other obstacle. $\nabla$

By Lemmas 7.2 and 7.3, if there exists a path between $q_{1}$ and $q_{2}$, then there exists a path between $H\left(q_{1}, 1\right)$ and $H\left(q_{2}, 1\right)$ and thus the rod-HGVG is connected.

\section{Conclusion}

This paper introduces a retract-like structure called the rod hierarchical generalized Voronoi graph. Although this structure was specifically developed for sensor based implementation of a rod, it can be used for classical motion planning as well. Moreover, since it is defined in terms of the distance function, the rod-HGVG readily lends itself to sensor based implementation. In fact, the incremental construction technique of the rodHGVG is similar to the incremental construction procedure for the point HGVG. Because of its graph-like structure, motion planning can be reduced to a onedimensional graph search. Simulations of this method are underway.

One of this method's limitations is that it assumes there are range sensors distributed throughout the body of the rod. Discrete sensor placements should adequately approximate such a sensor distribution, but this approximation is currently being investigated. Furthermore, there are environments where range sensor information cannot be readily provided, so a robot must rely on visual sensor data.

Since its definitions are based on those of the GVG, a rod-GVG for rods floating around in $\mathbb{R}^{3}$ (whose configuration space is $S E(3)$ ) can be readily defined. This extension is a future topic of research. The intention of this work is to provide a foundation for sensor based planning of robots modeled as convex sets.

\section{References}

[1] J.F. Canny and M.C. Lin. An Opportunistic Global Path Planner. Algorithmica, 10:102-120, 1993.

[2] H. Choset and J.W. Burdick. Sensor Based Planning and Nonsmooth Analysis. In Proc. IEEE Int. Conf. on Robotics and Automation, pages 3034-3041, San Diego, CA, 1994.

[3] H. Choset and J.W. Burdick. Sensor Based Planning, Part I The Generalized Voronoi Graph. In Proc. IEEE Int. Conf. on Robotics and Automation, Nagoya, Japan, 1995.

[4] H. Choset and J.W. Burdick. Sensor Based Planning, Part II Incremental Construction of the Generalized Voronoi Graph. In Proc. IEEE Int. Conf. on Robotics and Automation, Nagoya, Japan, 1995.

[5] 3. Cox and C.K. Yap. On-line Motion Planning: Case of a Planar Rod. Annals of Mathematics and Artifical Intelligence, 3:1-20, 1991.

[6] H.B. Keller. Lectures on Numerical Methods in Bifurcation Problems. Tata Institute of Fundamental Research, Bombay, India, 1987.
[7] V. Lumelsky and A. Stepanov. Path Planning Strategies for Point Mobile Automaton Moving Amist Unknown Obstacles of Arbitrary Shape. Algorithmica, 2:403-430, 1987.

[8] C. O'Dúnlaing and C.K. Yap. A "Retraction" Method for Planning the Motion of a Disc. Algorithmica, 6:104-111, 1985.

[9] M. O'Dúnlaing, C. Sharir and C.K. Yap. Generalized Voronoi Diagrams for Moving a Ladder. I: Topological Analysis. Communications on Pure and Applied Mathematics, 39:423-483, 1986.

[10] N. Rao, N.S.V. Stolzfus and S.S. Iyengar. A Retraction Method for Learned Navigation in Unknown Terrains for a Circular Robot. IEEE Transactions on Robotics and Au tomation, 7:699-707, October 1991.

[11] E. Rimon and J.F. Canny. Construction of C-space Roadmaps Using Local Sensory Data - What Should the Sensors Look For? In Proc. IEEE Int. Conf. on Robotics and Automation, pages 117-124, San Diego, CA, 1994.

[12] P. F. Rowat. "Representing the Spatial Experience and Solving Spatial Problems in a Simulated Robot Environment". In PhD. Thesis, University of British Columbia, 1979.

[13] Rao, N.S.V. Kareti, S. Shi, W. and Iyenagar, S.S. Robot Navigation in Unknown Terrains: Introductory Survey of Non-Heuristic Algorithms. Oak Ridge National Laboratory Technical Report, ORNL/TM-12410:1-58, July 1993.

Acknowledgements. The authors gratefully acknowledge the support of the Office of Naval Research, Grant \# N00014-93-1-0782. The authors profusely thank Dr. Andrew Lewis for his consistent help on our research. We also benefited greatly from conversations with Dr. Richard Murray, Luis Goncalves, William Law, and Scott Kelley.

\section{Appendix}

Proof of Proposition 5.1

Proof: For the first portion of the accessibility path, it was shown in [8] that there exists a path from an initial configuration to a configuration two-equidistant face, $\mathrm{eF}_{i j}$ by following $\nabla D_{i}(q)$ while maintaining a fixed orientation. Let $q_{1}$ be the configuration of the rod when it first becomes doubly equidistant. Hence, $D_{i}\left(q_{1}\right)=D_{j}\left(q_{1}\right)$ and $D_{i}\left(q_{1}\right)-D_{k}\left(q_{1}\right)<0$. Without loss of generality, assume that $q_{1}$ is not the closest configuration of the rod on $\mathrm{eF}_{i j}$ at the fixed orientation to objects $C_{i}$ and $C_{j}$. This way, $\pi_{T_{q_{1}}} \mathcal{F}_{\mathcal{F}_{i j}} \nabla D_{i} \neq 0$.

Now the second closest obstacle is $C_{k}\left(C_{i}\right.$ and $C_{j}$ are now both the first closest obstacles). Next, it needs to be shown that $\nabla D_{k}(q) \neq \nabla D_{i}(q)$. Assume $\nabla D_{k}(q)=$ $\nabla D_{i}(q)$. Since $D_{i}(q)<D_{k}(q), \nabla D_{k}=\nabla D_{i}$ implies that $C_{i}$ occludes $C_{k}$ which violates our original hypothesis that $C_{k}$ is always within line of sight of the rod. Hence, $\nabla D_{k}(q) \neq \nabla D_{i}(q),\left(\nabla D_{i}-\nabla D_{k}\right)(q) \neq 0$, and since $\nabla D_{i}$ and $\nabla D_{k}$ are both unit vectors, $\pi_{T_{q} \mathcal{E F}_{i j}}\left(\nabla D_{i}-\right.$ $\left.\nabla D_{k}\right)(q) \neq 0$ for all configurations $q$ on a path traced out by $\pi_{T_{q} \mathcal{F}_{i j}}\left(\nabla D_{i}-\nabla D_{k}\right)(q)$.

Since $\pi_{T_{q}} \mathcal{E F}_{i j}\left(\nabla D_{i}-\nabla D_{k}\right)(q) \neq 0$, by continuity of the distance function, gradient ascent following the gradient $\pi_{T_{q}} \mathcal{C F}_{i_{j}}\left(\nabla D_{i}-\nabla D_{k}\right)(q)$ traces a path from $q_{1}$ where $D_{i}\left(q_{1}\right)-D_{k}\left(q_{1}\right)<0$ to a configuration $q_{2}$ where 


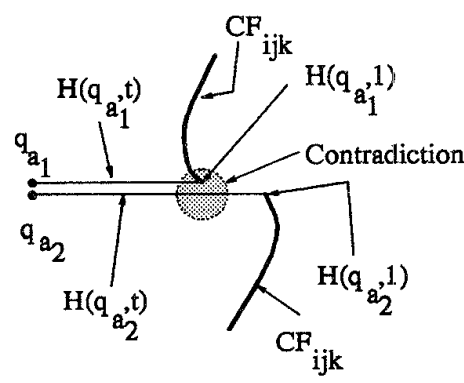

Fig. 12. The dark solid lines represent portions of $\mathcal{C} \mathcal{F}_{i j k}$. The thick straight lines represent the two accessibility paths of the two configurations $q_{a_{1}}$ and $q_{a_{2}}$ which start near each other. The shaded region represents the neighborhood of a point of $H\left(q_{a_{2}}, t\right)$ where the contradiction occurs.

$D_{i}\left(q_{2}\right)-D_{k}\left(q_{2}\right)=0 . q_{2}$ is a configuration in a rod-GVG edge because $D_{i}\left(q_{2}\right)=D_{j}\left(q_{2}\right)=D_{k}\left(q_{2}\right)$.

Proof of Corollary 5.2

Proof: For all $t \in[0, .5), D_{i}\left(H\left(q_{a}, t\right)\right)<D_{j}\left(H\left(q_{a}, t\right)\right)<$ $D_{k}\left(H\left(q_{a}, t\right)\right)<D_{h}\left(H\left(q_{a}, t\right)\right)$ for all $h \neq i, j, k$. By continuity of the distance function, there exists a neighborhood around each configuration $H\left(q_{a}, t\right)$ where $t \in[0, .5)$ such that for all configurations $q$ in this neighborhood, $D_{i}(q)<D_{j}(q)<D_{k}(q)<D_{h}(q)$ for all $h \neq i, j, k$. Therefore, there exists a tubular neighborhood surrounding the accessibility path described by $H$ from $q_{a}$ to $q_{b}$ such that

$$
D_{i}(q)<D_{j}(q)<D_{k}(q) \quad \forall h \neq i, j, k
$$

for all configurations in the neighborhood.

By a similar argument, there exists a similar tubular neighborhood from $q_{b}$ to $q_{c}$ such that for all $h \neq i, j, k$,

$$
\begin{gathered}
D_{i}(q) \leq D_{j}(q)<D_{k}(q)<D_{h}(q) \text { or } \\
D_{j}(q) \leq D_{i}(q)<D_{k}(q)<D_{h}(q)
\end{gathered}
$$

for all configurations in the second tube.

The proof now follows by contradiction. Assume that $H(q)$ is not continuous. Pick $q_{a_{1}}$ and $q_{a_{2}}$ such that $q_{a_{2}} \in$ $\operatorname{nbhd}\left(q_{a_{1}}\right)$ but $H\left(q_{a_{2}}, 1\right) \notin \operatorname{nbhd}\left(H\left(q_{a_{1}}, 1\right)\right)$ (i.e., $q_{c_{2}} \notin$ $\left.\operatorname{nbhd}\left(q_{c_{1}}\right)\right)$.

Since $H\left(q_{a_{2}}, 1\right) \notin \operatorname{nbhd}\left(H\left(q_{a_{1}}, 1\right)\right)$, there exists a $t<$ 1 for which either: (1) $H\left(q_{a_{1}}, 1\right) \in \operatorname{nbhd}\left(H\left(q_{a_{2}}, t\right)\right)$, or (2) $H\left(q_{a_{2}}, 1\right) \in \operatorname{nbhd}\left(H\left(q_{a_{1}}, t\right)\right)$. If the former were true, then there exists a $t<1$ for which there is a configuration, $q$, in the neighborhood of $H\left(q_{a_{2}}, t\right)$ where $D_{i}(q)=$ $D_{j}(q)=D_{k}(q)$. This contradicts the inequalities of Equations 13 and 14. If the $H\left(q_{a_{2}}, 1\right) \in \operatorname{nbhd}\left(H\left(q_{a_{1}}, t\right)\right)$, then a similar contradiction arises. Therefore, $H$ is continuous. Figure 12 helps visualize this proof.

\section{Proof of Proposition 6.1}

Proof: Assume without loss of generality that obstacles $C_{i}$ and $C_{j}$ have one unique pair of closest points, $c_{i}$ and $c_{j}$. Let the distance between these two points be $2 D_{\min }$. Therefore, for all points $c_{1} \in C_{i} \backslash\left\{c_{i}\right\}$, and for all points $c_{2} \in C_{j},\left\|c_{1}-c_{2}\right\|>2 D_{\min }$. This assumption implies that there exists a unique point, $r_{m i n} \in \mathcal{S}_{i j}$, where $d_{i}\left(r_{\min }\right)=d_{j}\left(r_{\min }\right)=D_{\min }$ and for all other points $r \in S S_{i j} \backslash\left\{r_{\min }\right\}, d_{i}(r)=d_{j}(r)>d_{i}\left(r_{\min }\right)=d_{j}\left(r_{\min }\right)$.

The proof follows in two steps. First, we show that for all configurations $q \in R \mathcal{F}_{i j}$ where $d_{i}(r)>D_{\min }$, there exists a unique configuration of the rod that is tangent to the point based GVG and that satisfies the inequalities in Equation 12. Second, we show that the set of configurations where $D_{i}(q)=D_{\min }$ forms a one dimensional curve in $S E(2)$.

Consider the case where $d_{i}(r)>D_{\min }$. Assume the point of contact $r$ is neither $q(P)$ nor $q(Q)$. By Equation $12, D_{i}(q)=d_{i}(r)$ which is greater than $D_{\text {min }}$, by hypothesis. Let the projection of the distance gradient at $r$ onto the rod be $\pi_{q(R)} \nabla d_{i}(r)$. We know $\pi_{q(R)} \nabla d_{i}(r)$ does not vanish because $D_{i}(q)>$ $D_{\text {min }}$. Hence, $-\pi_{q(R)} \nabla d_{i}(r) \neq 0$ and there exists a $y \in \operatorname{nbhd}(r) \bigcap q(R)$ such that $d_{i}(y)<d_{i}(r)$. This violates the inequality, $d_{i}(r) \leq d_{i}\left(r_{1}\right) \forall r_{1} \in q(R)$ (from Equation 12). Thus, the only points for which the rod may intersect $\mathcal{S S}_{i j}$ and maintain the inequality, $d_{i}(r) \leq d_{i}\left(r_{1}\right) \forall r_{1} \in q(R)$, is either $q(P)$ or $q(Q)$. Therefore, all configurations $g \in \mathcal{C S S}_{i j}$ that satisfy the inequality, $d_{i}(r) \leq d_{i}\left(r_{1}\right) \forall r_{1} \in q(R)$, can be identified with $S S_{i j} \times\{q(P)\}$ or $S S_{i j} \times\{q(Q)\}$, both of which are one-dimensional.

Now, consider the case where $d_{i}\left(r_{\min }\right)=d_{j}\left(r_{\min }\right)=$ $D_{\text {min }} . \pi_{q(R)} \nabla d_{i}\left(r_{\min }\right)$ vanishes for the set of configurations where $d_{i}\left(r_{\text {min }}\right)=d_{j}\left(r_{\text {min }}\right)=D_{\text {min }}$. Thus, for all configurations of the the rod where $r_{\min } \bigcap q(R)=r_{\min }$, there always exists a neighborhood, $\operatorname{nbhd}\left(r_{\min }\right) \bigcap q(R)$, where $d_{i}(y) \geq d_{i}\left(r_{\min }\right)$ for all $y \in \operatorname{nbhd}\left(r_{\min }\right) \bigcap q(R)$. Therefore, all such configurations can be identified with $\left\{r_{\min }\right\} \times[0, L]$ which is also one-dimensional.

The inequality $d_{i}(r) \leq D_{h}(q) \forall h \neq i, j$ forces the rod to be closest to obstacles $C_{i}$ and $C_{j}$, but does not affect the dimensionality of the edges.

\section{Proof of Lemma 6.3}

Proof: Recall that for all configurations $q \in \mathcal{R}_{i j}$, there exists an $r \in q(R)$ such that $d_{i}(r) \leq d_{i}\left(r_{1}\right)$, and $d_{j}(r) \leq d_{j}\left(r_{1}\right)$ for all points $r_{1} \in q(R)$. Since $D_{i}(q)=$ $\min _{r \in R(q), c \in C_{i}}\|r-c\|, d_{i}(r)=D_{i}(q)$ and $d_{j}(r)=D_{j}(q)$. Therefore for all configurations $q \in \mathcal{R}_{i j}, D_{i}(q)=D_{j}(q)$ and thus for all $q \in \mathcal{R}_{i j}, q \in \mathcal{C F}_{i j}$.

\section{Proof of Lemma 6.4}

Proof: By definition, for all $q \in \mathcal{R}_{i j}$, there exists $r \in q(R)$ such that $d_{i}\left(r_{1}\right) \geq d_{i}(r)$ for all $r_{1} \in q(R)$. Since $d_{i}(r) \geq 0$, for all $r_{1} \in q(R), d_{i}\left(r_{1}\right)>0$ because we assume the rod does not fully intersect an obstacles boundary. Thus, with perhaps the exception of the point $P$ or $Q$, the rod does not intersect an obstacle. 